\title{
Comparison of genome-wide array genomic hybridization platforms for the detection of copy number variants in idiopathic mental retardation
}

\author{
Tracy Tucker ${ }^{1 *}$, Alexandre Montpetit ${ }^{2}$, David Chai ${ }^{3}$, Susanna Chan ${ }^{4}$, Sébastien Chénier ${ }^{5}$, Bradley P Coe ${ }^{6}$, \\ Allen Delaney ${ }^{4}$, Patrice Eydoux ${ }^{3}$, Wan L Lam ${ }^{6}$, Sylvie Langlois ${ }^{3,7}$, Emmanuelle Lemyre ${ }^{5}$, Marco Marra ${ }^{4}$, Hong Qian ${ }^{4}$, \\ Guy A Rouleau ${ }^{8,9}$, David Vincent ${ }^{2}$, Jacques L Michaud ${ }^{5,8}$ and Jan M Friedman ${ }^{1,7}$
}

\begin{abstract}
Background: Clinical laboratories are adopting array genomic hybridization as a standard clinical test. A number of whole genome array genomic hybridization platforms are available, but little is known about their comparative performance in a clinical context.

Methods: We studied 30 children with idiopathic MR and both unaffected parents of each child using Affymetrix 500 K GeneChip SNP arrays, Agilent Human Genome 244 K oligonucleotide arrays and NimbleGen 385 K WholeGenome oligonucleotide arrays. We also determined whether CNVs called on these platforms were detected by Illumina Hap550 beadchips or SMRT $32 \mathrm{~K} \mathrm{BAC}$ whole genome tiling arrays and tested 15 of the 30 trios on Affymetrix 6.0 SNP arrays.

Results: The Affymetrix 500 K, Agilent and NimbleGen platforms identified 3061 autosomal and 117 X chromosomal CNVs in the 30 trios. 147 of these CNVs appeared to be de novo, but only 34 (22\%) were found on more than one platform. Performing genotype-phenotype correlations, we identified 7 most likely pathogenic and 2 possibly pathogenic CNVs for MR. All 9 of these putatively pathogenic CNVs were detected by the Affymetrix 500 K, Agilent, NimbleGen and the Illumina arrays, and 5 were found by the SMRT BAC array. Both putatively pathogenic CNVs identified in the 15 trios tested with the Affymetrix 6.0 were identified by this platform.

Conclusions: Our findings demonstrate that different results are obtained with different platforms and illustrate the trade-off that exists between sensitivity and specificity. The large number of apparently false positive CNV calls on each of the platforms supports the need for validating clinically important findings with a different technology.
\end{abstract}

\section{Background}

Chromosomal abnormalities, the most frequently diagnosed cause of mental retardation (MR) [1], are routinely identified by cytogenetic analysis. Studies using array genomic hybridization (AGH) have found apparently-pathogenic gains or losses of genetic material in at least $10 \%$ of children with MR and normal conventional cytogenetic analysis [2-4]. These apparently pathogenic deletions and duplications range in size from $<100 \mathrm{~Kb}$ to $15 \mathrm{Mb}$. Such submicroscopic chromosomal gains or

\footnotetext{
* Correspondence: tbtucker@interchange.ubc.ca

'Department of Medical Genetics, University of British Columbia, Vancouver, British Columbia, Canada

Full list of author information is available at the end of the article
}

losses are collectively called pathogenic copy number variants (CNVs).

However, most CNVs, despite producing genomic imbalance of many thousands of DNA base pairs, do not cause MR. In fact, CNVs are the greatest source of genetic variation in normal people; the mean number of apparently benign CNVs observed ranges from 10's1000 's per person, depending on the technology used [5-9]. Distinguishing benign CNVs from those that cause MR and other birth defects is the most serious challenge to the routine clinical use of AGH, especially for prenatal diagnosis $[4,10-13]$.

A consensus has developed that AGH should be offered routinely in the evaluation of children with MR

\section{() Biomed Central}


and other birth defects $[2,3,11]$. However, there is no agreement regarding the choice of AGH platform, resolution, or reference sample that is most appropriate for clinical use [3,14-17]. AGH for clinical diagnosis has often employed targeted arrays with probes in genomic regions known to be associated with microdeletion and microduplication syndromes, and more recent versions of many targeted arrays have added additional probes (i.e., a 'backbone') to provide some degree of genomewide coverage. As the density of probes in these backbones increases, genome-wide and targeted platforms are converging, with both providing a survey of the whole genome at relatively high resolution.

Few studies have compared AGH whole genome technologies [18-20], and most are retrospective and limited to pathogenic CNVs. We performed AGH studies on $30 \mathrm{MR}$ trios (children with idiopathic MR and both of their unaffected parents) using three different high-resolution genome-wide oligonucleotide platforms - Affymetrix $500 \mathrm{~K}$, Agilent $244 \mathrm{~K}$ and NimbleGen $385 \mathrm{~K}$ - to assess their utility for the identification of pathogenic CNVs in children with MR. We determined whether the CNVs called on these platforms were also detected by the Illumina Hap550 Beadchip or the SMRT $32 \mathrm{~K} \mathrm{BAC}$ whole genome tiling array and tested samples from 15 of the MR trios on Affymetrix 6.0 arrays. This large comparison of multiple AGH platforms provides unique insights into both the power and the limitations of current technology for detecting pathogenic genomic imbalance in children with MR.

\section{Methods \\ Patients}

Patients with MR and at least one of the following additional characteristics were selected for study: 1) growth retardation of pre- and/or post-natal onset; 2) microcephaly or macrocephaly; 3 ) one or more major malformations, and 4) more than two facial dysmorphic features. Characterization of this cohort with the checklist developed by de Vries et al. [21] showed an average score of 4.4 (range: 2-9). The cause of the MR in each child was unknown despite full evaluation by a clinical geneticist, a karyotype at $\geq 500$ band resolution and subtelomeric FISH studies. This study was approved by the University of British Columbia Clinical Research Ethics Board and Hopital Sainte-Justine Research Ethics Board, and informed consent was obtained from each family.

\section{Array Genomic Hybridization}

Genomic DNA was extracted from blood samples using the Puregene DNA kit (Gentra System). DNA quality for each trio was assessed by electrophoresis in a $1 \%$ agarose gel, and DNA concentration was measured with a NanoDrop ${ }^{\mathrm{TM}}$ Spectrophotometer.
AGH was performed on 30 children with idiopathic MR and on both normal parents of each child on Affymetrix $500 \mathrm{~K}$ GeneChips, Agilent $244 \mathrm{~K}$ Oligonucleotide Arrays, and NimbleGen 385 K Oligonucleotide Arrays. In addition, DNA from the 30 children was run on Illumina Hap550 Beadchips using a set of about 100 HapMap samples as reference. DNAs from the 30 children were also run on Sub-Megabase Resolution Tiling-set (SMRT) human genomic BAC arrays using one of the parents - the one of the same sex - as reference. In addition, DNA samples from 15 trios were run on the Affymetrix Genome-Wide Human SNP Array 6.0.

All samples were handled according to the platform manufacturer's recommendations, and CNV detection was performed using the manufacturer's recommended software with default settings (See Additional File for detailed protocol and software settings).

\section{Identification of Autosomal de novo Changes}

A CNV was considered to be de novo on Affymetrix 500 K, Agilent 244 K, NimbleGen 385 K, or Affymetrix 6.0 AGH if a set of probes identified by the platform algorithm was called as a deletion in the child relative to both parents or as a duplication in the child relative to both parents on the same platform.

\section{Identification of X Chromosomal CNVs}

In addition to looking for de novo CNVs as described for the autosomes above, we performed manual assessments for CNVs when there was a sex-mismatch between the child and parent because the NimbleGen and Affymetrix platforms are unable to account for sex mismatches between the test and reference DNAs.

\section{Identification of Pathogenic Changes}

We used de novo occurrence as a major criterion of pathogenicity for autosomal CNVs; however, most de novo changes found in these studies are unlikely to be pathogenic for MR in the children studied. In addition, all X chromosomal CNVs except those in the pseudoautosomal or XY homology regions were assessed for pathogenicity. We used previously published criteria $[4,12,13,22,23]$ to determine which CNVs are likely to be pathogenic. We performed genotype-phenotype correlations only on CNVs that were greater than $50 \mathrm{~Kb}$ in length and that met the criteria of pathogenicity cited above.

\section{CNV Confirmation}

CNVs were validated by FISH, MLPA, qPCR or PCR (for $\mathrm{X}$ chromosome deletions identified in males). See Additional File 1 for detailed validation protocols. 


\section{Statistical Analysis}

A chi square analysis was performed to assess differences between platforms in the proportion of singleton $\mathrm{CNV}$ calls and the number of de novo CNVs. Differences in sizes and types of CNVs called between platforms were assessed by Mann-Whitney U tests. A p-value of 0.05 was considered significant in all analyses.

\section{Results}

We compared the ability of the Affymetrix $500 \mathrm{~K}$, Agilent $244 \mathrm{~K}$ and NimbleGen $385 \mathrm{~K}$ platforms to detect de novo CNVs in 30 patients with idiopathic MR using the normal parents of each child as reference.

Overall, 1,492 autosomal deletions and 1,569 autosomal duplications were called in the 30 children with MR by one or more of the three main platforms (Affymetrix $500 \mathrm{~K}$, Agilent $244 \mathrm{~K}$, and NimbleGen $385 \mathrm{~K}$; Additional File 2 and Additional File 1). Over 80\% of the autosomal $\mathrm{CNV}$ calls were made by only one of the three major platforms. The proportion of singleton CNV calls made was significantly different among the 3 platforms $(\mathrm{p}=<$ 0.001). The NimbleGen platform identified about $40 \%$ more autosomal calls than the Agilent platform, while the higher density Affymetrix $500 \mathrm{~K}$ platform identified fewer than one third as many CNVs as the Agilent platform (Figure 1). However, $60 \%$ of the autosomal CNVs identified only on the Agilent or NimbleGen platform are in genomic regions that had fewer than 5 probes on the Affymetrix $500 \mathrm{~K}$ array, so recognition of such CNVs would not be expected.

\section{Detection of autosomal de novo CNVs}

There were 146 autosomal de novo CNVs identified on one or more of the three main platforms in the $30 \mathrm{MR}$ probands, an average of 4.9 de novo CNVs per patient

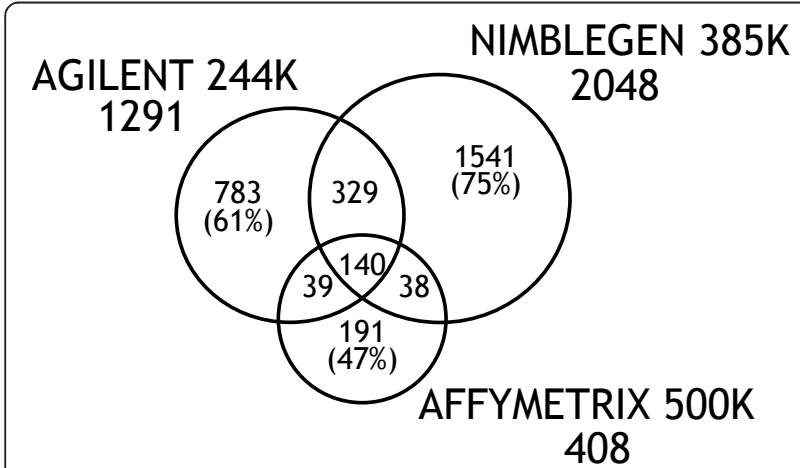

Figure 1 Venn diagrams of CNV calls made by the 3 main AGH platforms. The numbers under each platform name indicate the total number of CNV calls by that platform. The numbers in the intersecting regions indicate CNV calls made by multiple platforms. The numbers outside the intersecting regions are the number of CNVs that were unique to that platform.
(Additional File 3). Two patients had only one de novo CNV identified, 3 had 2 de novo CNVs, 5 had 3 de novo CNVs and 20 patients had 4 or more de novo CNVs.

$114(78 \%)$ of the autosomal de novo calls were identified on only one platform, 23 on 2 platforms and 9 on all 3 platforms (Figure 2). Significantly fewer de novo calls were made with the Affymetrix $500 \mathrm{~K}$ system than with Agilent $(\mathrm{p}=<0.001)$ or NimbleGen platforms $(\mathrm{p}=<0.001)$.

A larger number of autosomal de novo deletions (96) than duplications (50) were called. The median size of the de novo deletions $(113 \mathrm{~Kb})$ was similar to that of the de novo duplications $(98 \mathrm{~Kb})(\mathrm{p}=0.348)$. The median size of the de novo CNVs detected by the NimbleGen platform $(183 \mathrm{~Kb})$ was significantly larger than those identified by the Agilent platform $(82 \mathrm{~Kb} ; \mathrm{p}=0.003)$ but not significantly larger than those identified by the Affymetrix $500 \mathrm{~K}$ platform $(145 \mathrm{~Kb} ; \mathrm{p}=0.083$; Additional File 1).

Many autosomal de novo CNVs were observed in multiple individuals in this small series. These recurrent calls accounted for 45 (31\%) of the 146 autosomal de novo CNVs called in the 30 trios, and all occurred in regions that contain polymorphic CNVs previously recognized by oligonucleotide arrays or higher resolution techniques (Database of Genomic Variants (DGV), http://projects.tcag.ca/variation/) (Additional File 3).

\section{Detection of autosomal de novo CNVs with the Affymetrix 6.0 Platform}

To explore whether the higher density Affymetrix 6.0 array improved CNV detection in comparison to the three main platforms, we arbitrarily selected 15 of the $30 \mathrm{MR}$ trios for analysis using the Affymetrix 6.0 platform (see Additional File 1 for details of analysis).

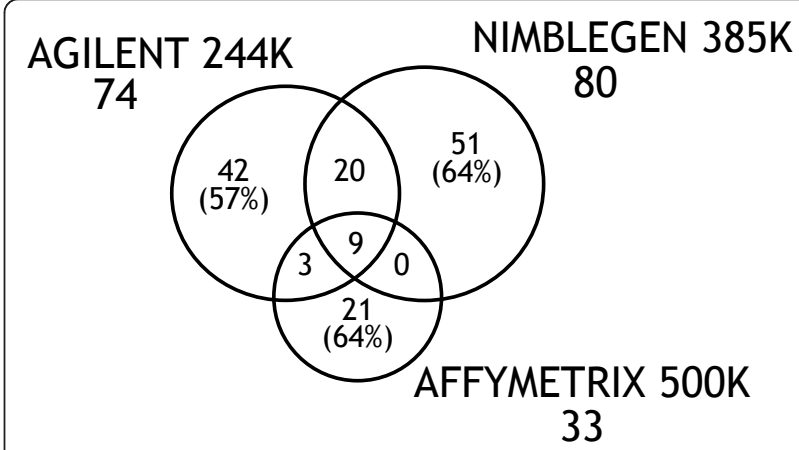

Figure 2 Venn diagrams of autosomal de novo CNV calls made by the $\mathbf{3}$ main AGH platforms. The numbers under each platform name indicate the total number of de novo CNV calls by that platform. The numbers in the intersecting regions present CNV calls made by multiple platforms. The numbers outside the intersecting regions are the number of CNVs that were unique to that platform. 
There was a total of 915 autosomal CNVs identified with the Affymetrix 6.0 platform in the 15 probands (an average of 61 CNVs per person) (Additional File 4), compared to 281 autosomal CNVs (an average of 18.7 per person) in these same 15 individuals on the Affymetrix $500 \mathrm{~K}$ platform. $682 \mathrm{CNVs}$ were called in these 15 probands on the Agilent platform and $973 \mathrm{CNVs}$ were called on the NimbleGen platform.

41 of the 915 autosomal CNVs identified by Affymetrix 6.0 AGH occurred de novo, and 29 of these 41 (71\%) CNVs were not found by any of the three main platforms. The Affymetrix 6.0 platform identified 9 of $12 \mathrm{CNVs}$ that were called as de novo on 2 or 3 of the main platforms in these patients (Additional File 3).

\section{Detection of autosomal de novo CNVs with the Illumina and SMRT Platforms}

In more limited comparisons, we sought to determine whether autosomal de novo CNVs identified by the three main platforms studied are likely to be identified by the Illumina Hap550 Beadarray or the SMRT $32 \mathrm{~K}$ BAC tiling array.

The Illumina platform identified 14 of the 146 autosomal de novo CNVs that had been called on one or more of the 3 main platforms and 8 of the 9 de novo CNVs that had been called on all 3 main platforms (Additional File 3). All Illumina CNV calls are listed in Additional File 5.

The SMRT platform identified 9 of the 146 autosomal de novo CNVs called on one or more of the 3 main platforms, including 4 of the 9 de novo CNVs called on all 3 of the main platforms (size range: $333 \mathrm{~Kb}-9.8 \mathrm{Mb}$ ). All CNVs called by SMRT AGH are listed in Additional File 6.

\section{Detection of $\mathrm{X}$ chromosomal CNVs}

The X chromosome was analysed separately because half of our hybridizations are sex-mismatched and the Affymetrix and NimbleGen CNV detection software are unable to correct for this. Therefore, identifying CNVs in sex-mismatched hybridizations on these platforms required manual assessment, a process that is inherently more subjective than the automated assessment used for the other platforms.

There were $117 \mathrm{X}$-chromosomal CNVs identified on one or more of the 3 main platforms in these $30 \mathrm{MR}$ trios (Additional File 7). 23 of these 117 CNVs were identified in 9 females. 101 (86\%) of the CNVs were identified by only 1 platform (Figure 3 ).

Fewer X-chromosomal CNV calls were made on the Agilent (38) and Affymetrix $500 \mathrm{~K}$ (26) platforms than on the NimbleGen platform (73). A larger number of deletions (78) than duplications (39) were identified on the $\mathrm{X}$ chromosome. The median size of the deletions

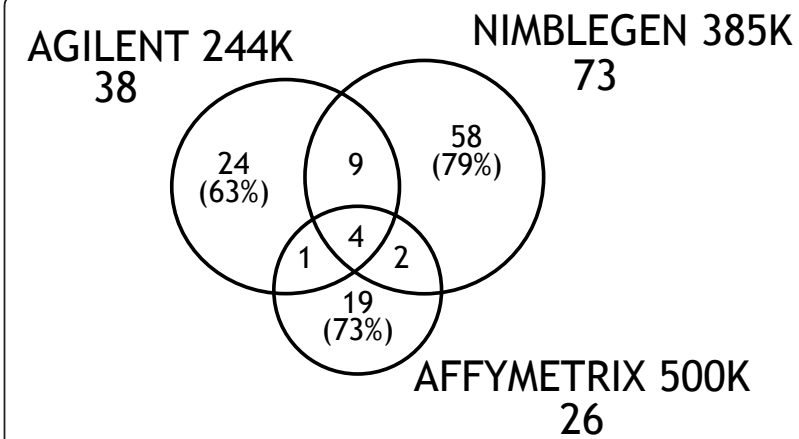

Figure 3 Venn diagram of X chromosome CNV calls made by the $\mathbf{3}$ main AGH platforms. The numbers under each platform name indicate the total number of CNV calls by that platform. The numbers in the intersecting regions present CNV calls made by multiple platforms. The numbers outside the intersecting regions are the number of CNVs that were unique to that platform.

$(131 \mathrm{~Kb})$ was similar to that of the duplications $(97 \mathrm{~Kb}$, $\mathrm{p}=0.175)$. The median size of the $\mathrm{X}$-chromosomal CNVs detected by the Agilent platform (32 Kb) was significantly smaller than that of the CNVs identified by the Affymetrix $500 \mathrm{~K}(209 \mathrm{~Kb}, \mathrm{p}<0.001)$ or NimbleGen (168 Kb, $\mathrm{p}<0.001)$ platforms.

Four of the $117 \mathrm{X}$-chromosomal CNVs called on the 3 main platforms were also identified by the Illumina platform, 6 by the Affymetrix 6.0 platform and 1 by the SMRT BAC platform (Additional File 7).

\section{Autosomal de novo CNVs with Potential Clinical Significance}

In a clinical laboratory, it is not usually possible to use multiple platforms to determine which CNV calls are real, and only a subset of the CNVs called with any technology is likely to be pathogenic. We used previously published criteria to determine which CNVs identified by one or more of the Agilent, NimbleGen, Affymetrix $500 \mathrm{~K}$ and Affymetrix 6.0 platforms are likely to be pathogenic (Additional File 4).

Only autosomal CNVs that occurred de novo were assessed for pathogenicity. Although inherited CNVs may cause MR [24-27], they are much less likely to do so than de novo CNVs, and there was no clinical reason to suspect a pathogenic inherited $\mathrm{CNV}$ in any of these children.

Smaller CNVs are much more likely than large CNVs to be false positives and to be benign rather than pathogenic [23]. Therefore, we restricted the analysis for likely pathogenicity to de novo CNVs that were $50 \mathrm{~Kb}$ or larger. This eliminated from consideration 51 CNVs (36 deletions and 15 amplifications) that were $<50 \mathrm{~Kb}$.

We also eliminated from consideration 21 de novo CNVs (13 deletions and 8 duplications) that did not contain validated open reading frames because such 
CNVs cannot be interpreted as pathogenic unless they involve a non-coding region known to be associated with MR. We eliminated a further 30 de novo CNVs (20 deletions and 10 duplications) that occurred in regions that contain only genetically unstable highly repetitive genes such as olfactory receptor genes or immunoglobin genes. In addition, given the small sample size, any de novo CNV that was identified in 4 or more probands with differing phenotypes and that was not known to be pathogenic for MR was deemed unlikely to be pathogenic, and we eliminated an additional 19 CNVs (8 deletions and 11 duplications) for this reason.

We subjected 21 of the remaining 25 de novo CNVs identified in 14 individuals to FISH or MLPA validation. Eight of these de novo CNVs were called by all 3 of the main platforms, and all 8 were confirmed by FISH or MLPA. In contrast, the 11 de novo CNVs identified on just one of the 3 main platforms and two de novo CNVs identified by two of the 3 main platforms could not be validated by FISH or MLPA.

\section{Chromosomal CNVs with Potential Clinical Significance}

We considered 31 of the $117 \mathrm{X}$-chromosomal CNVs that were less than $50 \mathrm{~Kb}$ and 21 that did not contain open reading frames unlikely to be pathogenic and eliminated them from further assessment. 32 other X- chromosomal CNVs were recurrent within this study population and were also eliminated from further analysis. One $7 \mathrm{Mb}$ de novo amplification in a male was validated by FISH (Patient 8960) and was identified by all 3 of the main platforms. 17 other CNVs were tested with PCR or qPCR and could not be confirmed (Additional File 1). These 17 CNVs were eliminated from further consideration as being pathogenic.

\section{Genotype-Phenotype Correlations}

Of the 28 remaining de novo CNVs, 12 were autosomal and 16, X-linked. We considered 4 autosomal de novo CNVs (Table 1) and $11 \mathrm{X}$ chromosomal CNVs (Table 2) to be unlikely to cause MR because they contained no RefSeq genes that appeared to be reasonable candidates for pathogenicity. Two X-chromosomal CNVs result in a MR phenotype in males but not in females $[28,29]$, and both were eliminated from further consideration because they occurred in a female (Patient 7093). One X chromosomal deletion in a male (Patient 3094) was eliminated because it results in MR when deleted in females but not in males [30]. Another maternally inherited duplication was eliminated in a female (Patient 1815) because only deletions have been reported to result in MR in females [31].

Table 1 Summary of autosomal de novo CNVs identified on the three main AGH platforms selected for genotypephenotype analysis

\begin{tabular}{ccccccccc}
\hline $\begin{array}{c}\text { Trio } \\
\text { ID }\end{array}$ & Chr & $\begin{array}{c}\text { CNV } \\
\text { Type }\end{array}$ & Start* & Size* & $\begin{array}{c}\text { Platforms } \\
\text { Identified CNV }\end{array}$ & $\begin{array}{c}\text { \# of RefSeq } \\
\text { Genes }\end{array}$ & Validation & Comment on Gene Function \\
\hline 1815 & 3 & DEL & 196904149 & 54518 & Agilent & 1 & NT & $\begin{array}{c}\text { Mucin 20 - Expression pattern not consistent } \\
\text { with causing MR [40] }\end{array}$ \\
\hline 4821 & 5 & DEL & 68950015 & 1329642 & NimbleGen & 7 & NT & $\begin{array}{c}\text { Mutations in SMN1 associated with spinal } \\
\text { muscle atrophy [41] }\end{array}$ \\
\hline 8960 & 5 & DUP & 180309941 & 55922 & NimbleGen & 2 & MLPA Pos & $\begin{array}{c}\text { Expression pattern not consistent with causing } \\
\text { MR [42] }\end{array}$ \\
\hline 1815 & 6 & DEL & 111807663 & 9889630 & All 3 platforms & 57 & FISH Pos & Likely pathogenic based on size \\
\hline 7531 & 9 & DEL & 139496489 & 333935 & All 3 platforms & 7 & FISH Pos & $\begin{array}{c}\text { CNVs in region previously reported as } \\
\text { pathogenic [32] }\end{array}$ \\
\hline 1815 & 12 & DEL & 11371263 & 83667 & NimbleGen & 1 & MLPA Pos & $\begin{array}{c}\text { Expression pattern not consistent with causing } \\
\text { MR [43] }\end{array}$ \\
\hline 1056 & 13 & DEL & 107190506 & 2206948 & All 3 platforms & 5 & FISH Pos & $\begin{array}{c}\text { Encompassed within de novo CNV in DECIPHER } \\
\text { patient with MR }\end{array}$ \\
\hline 4821 & 16 & DEL & 3862993 & 78891 & All 3 platforms & 1 & MLPA Pos & $\begin{array}{c}\text { CNVs in region previously reported as } \\
\text { pathogenic [35] }\end{array}$ \\
\hline 3921 & 17 & DEL & 41062469 & 657364 & All 3 platforms & 8 & FISH Pos & $\begin{array}{c}\text { CNVs in region previously reported as } \\
\text { pathogenic [33] }\end{array}$ \\
\hline 9609 & 21 & DEL & 33902218 & 152885 & All 3 platforms & 2 & MLPA Pos & Important in spinal development [37] \\
\hline 9609 & 22 & DEL & 19062809 & 728798 & All 3 platforms & 19 & FISH Pos & $\begin{array}{c}\text { CNVs in region previously reported as } \\
\text { pathogenic [34,44] }\end{array}$ \\
\hline 8327 & 22 & DUP & 19412033 & 378797 & All 3 platforms & 13 & MLPA Pos & $\begin{array}{c}\text { Mutation has been reported in family with } \\
\text { normal phenotype [25] }\end{array}$ \\
\hline
\end{tabular}

$\mathrm{DEL}=$ Deletion; DUP = Amplification, NT - Not tested; Pos = Positive; Neg = Negative; N/T = Not tested.

*Start/end coordinates determined from largest region of overlap between any two platforms; size is the difference between these two coordinates (Build 36). 
Table 2 Summary of $X$ chromosome CNVs identified on the three main AGH platforms selected for genotypephenotype analysis

\begin{tabular}{|c|c|c|c|c|c|c|c|}
\hline $\begin{array}{l}\text { Trio } \\
\text { ID }\end{array}$ & $\begin{array}{l}\text { CNV } \\
\text { Type }\end{array}$ & Start $^{*}$ & Size* & $\begin{array}{c}\text { Platforms } \\
\text { Identified CNV }\end{array}$ & Genes Involved & Validation & Comment on Gene Function \\
\hline 6428 & DEL & 148264112 & 156992 & Affy & IDS & NT & $\begin{array}{c}\text { Expression pattern not consistent with } \\
\text { causing MR }\end{array}$ \\
\hline 2894 & DEL & 101266713 & 250045 & NimbleGen & 5 RefSeq genes & NT & $\begin{array}{c}\text { Expression pattern not consistent with } \\
\text { causing MR [45] }\end{array}$ \\
\hline 3519 & DEL & 9454329 & 197920 & Affy & TBL1X & NT & $\begin{array}{c}\text { Expression pattern not consistent with } \\
\text { causing MR [46] }\end{array}$ \\
\hline 8960 & DUP & 67416262 & 7057217 & All 3 platforms & 57 RefSeq genes & FISH POS & FISH POS \\
\hline 9313 & DEL & 9484049 & 165559 & Affy & TBL1X & NT & $\begin{array}{c}\text { Expression pattern not consistent with } \\
\text { causing MR }\end{array}$ \\
\hline 3921 & DUP & 74811330 & 208698 & NimbleGen & $\pi \mathrm{TC3L}$ & NT & $\begin{array}{l}\text { Expression pattern not consistent with } \\
\text { causing MR [47] }\end{array}$ \\
\hline 1511 & DEL & 6856649 & 201556 & Affy & HDHD1A & NT & $\begin{array}{l}\text { Expression pattern not consistent with } \\
\text { causing MR [48] }\end{array}$ \\
\hline 2714 & DEL & 76534899 & 67182 & NimbleGen & FGF16 & NT & $\begin{array}{c}\text { Expression pattern not consistent with } \\
\text { causing MR [49] }\end{array}$ \\
\hline 5993 & DEL & 6932549 & 130699 & NimbleGen & HDHD1A & NT & $\begin{array}{l}\text { Expression pattern not consistent with } \\
\text { causing MR [48] }\end{array}$ \\
\hline 4821 & DEL & 6625133 & 419230 & NimbleGen & HDHD1A & NT & $\begin{array}{c}\text { Expression pattern not consistent with } \\
\text { causing MR [48] }\end{array}$ \\
\hline 3921 & DUP & 74811330 & 208698 & NimbleGen & MAGEE2 & NT & $\begin{array}{c}\text { Expression pattern not consistent with } \\
\text { causing MR [47] }\end{array}$ \\
\hline 8960 & DEL & 29967317 & 203809 & Affy & MEGB2E & NT & $\begin{array}{l}\text { Expression pattern not consistent with } \\
\text { causing MR [50] }\end{array}$ \\
\hline 1815 & DUP & 67767923 & 2019581 & NimbleGen & $\begin{array}{l}15 \text { RefSeq genes } \\
\text { including DLG3 }\end{array}$ & NT & $\begin{array}{c}\text { Amplification not reported to cause MR } \\
{[31]}\end{array}$ \\
\hline 7093 & DUP & 73429587 & 263642 & NimbleGen & $\begin{array}{l}3 \text { RefSeq genes including } \\
\text { SLC16A2 }\end{array}$ & NT & Females not affected [28] \\
\hline 3094 & DEL & 99293227 & 205443 & Affy & PCDH19 & NT & Males not affected [30] \\
\hline 7093 & DEL & 6687308 & 906505 & $\begin{array}{l}\text { Agilent \& } \\
\text { NimbleGen }\end{array}$ & STS & NT & Females not affected [29] \\
\hline
\end{tabular}

DEL = Deletion; DUP = Amplification, NT - Not tested; Pos = Positive; Neg = Negative; N/T = Not tested.

*Start/end coordinates determined from largest region of overlap between any two platforms; size is the difference between these two coordinates (Build 36).

Of the remaining 9 de novo CNVs, 7 are likely to be pathogenic. Table 3 summarizes the phenotypes of these patients. The CNVs that are likely to be pathogenic include deletions within 9q34.3 (Patient 7531), 17q21.31 (Patient 3921) and 22q11.2 (Patient 9609) that have been previously reported to be pathogenic in other patients with similar phenotypes [32-34]. A 9.8 MB deletion (Patient 1815) and $7 \mathrm{Mb}$ duplication (Patient 8960 ) are likely to be pathogenic based on their size and the number of genes affected. Patient 4821 has a $78 \mathrm{~Kb}$ deletion within the first exon and upstream sequence of the CREB binding protein (CREBBP), haploinsufficiency of which causes the Rubinstein-Taybi syndrome [35]. The phenotype of Patient 4821 is consistent with this diagnosis. The seventh patient has a previously undescribed 2.2 Mb deletion of chromosome 13q11 (Patient 1056) that encompasses 5 genes, including myosin 16 (MYO16), which codes for a protein that interacts with known synaptic proteins that are important for cognition [36]. The deletion in this patient falls within a 9.9 $\mathrm{Mb}$ de novo deletion in another patient with $\mathrm{MR}$ who is listed in DECIPHER (DECIPHER patient ID 4668).

These 7 de novo CNVs that are likely to be pathogenic were all identified on all three of the main AGH platforms studied - Agilent, NimbleGen and Affymetrix 500 $\mathrm{K}$ - as well as on the Illumina platform. Five of these 7 cases were also identified by SMRT AGH (Additional Files 3 and 7), and the Affymetrix 6.0 platform detected the only CNV tested on that platform that is very likely to be pathogenic (Patient 4821). None of the de novo CNV calls made only on the Affymetrix 6.0 platform occurred in regions that are known to be pathogenic for MR.

Two other validated de novo CNVs may be pathogenic. The first is a $152 \mathrm{~Kb}$ deletion of chromosome 21 that involves intersectin 1 , which regulates endocytosis and dendritic spine development [37]. This deletion 
Table 3 Summary of phenotypes in patients with validated pathogenic or possibly pathogenic CNVs

\begin{tabular}{|c|c|c|c|c|c|c|}
\hline ID & Chr & CNV & Start $^{*}$ & Size* & Pathogenicity & Phenotype \\
\hline 1815 & 6 & DEL & 111807663 & 9889630 & Likely & $\begin{array}{l}\text { MR, microcephaly, epicanthic folds, small ears, hypoplastic lobes, micrognathia, } \\
\text { brachycephaly, hypotonia }\end{array}$ \\
\hline 7531 & 9 & DEL & 139496489 & 333935 & Likely & $\begin{array}{c}\text { Moderate global developmental delay, microcephaly, flat face, upslanting palpebral } \\
\text { fissures, hypertelorism, synophrysm, anteverted nares, hypoplasia of the } \\
\text { amygdalo-hippocampic complex }\end{array}$ \\
\hline 1056 & 13 & DEL & 107190506 & 2206948 & Likely & Moderate MR, upslanting palpebral fissures, retrognathia \\
\hline 4821 & 16 & DEL & 3862993 & 78891 & Likely & $\begin{array}{c}\text { Moderate MR, microcephaly, short stature, bilateral glaucoma, bilateral } \\
\text { colobomas of the optic nerves, neuro-sensorial deafness, large ASD, epicanthic } \\
\text { folds, low nasal septum, preauricular pits, low set ears, broad distal phalanges } \\
\text { of all fingers and toes, cryptorchidy, hypotonia }\end{array}$ \\
\hline 3921 & 17 & DEL & 41062469 & 657364 & Likely & $\begin{array}{l}\text { Mild MR, sagittal craniosynostosis, malar hypoplasia, mild retrognathia, short } \\
\text { and upslanting palpebral fissures, low set ears, high arched palate, broad } \\
\text { proximal phalangeal joints of the hands, unilateral cryptorchidism }\end{array}$ \\
\hline 9609 & 22 & DEL & 19062809 & 728798 & Likely & $\begin{array}{l}\text { Moderate MR, microcephaly, short stature, down-slanting palpebral fissures, } \\
\text { low-set ears, wide nasal base, retrognathia, metopic craniosynostosis, cleft } \\
\text { palate, partial agenesis of the corpus callosum, tetralogy of Fallot }\end{array}$ \\
\hline 8960 & $x$ & DUP & 67416262 & 7057217 & Likely & $\begin{array}{c}\text { Moderate MR, brachycephaly, bilateral epicanthic folds, posteriorly rotated } \\
\text { ears with hypoplastic helix and hypotnic }\end{array}$ \\
\hline 9609 & 21 & DEL & 33902218 & 152885 & Possible & See above pathogenic mutation \\
\hline 8327 & 22 & DUP & 19412033 & 378797 & Possible & Mild MR, small stature, Pierre Robin sequence with cleft palate \\
\hline
\end{tabular}

$\mathrm{DEL}=$ Deletion; $\mathrm{DUP}=$ Amplification .

*Start/end coordinates determined from largest region of overlap between any two platforms; size is the difference between these two coordinates (Build 36).

occurred in Patient 9609, who also has a pathogenic 728 $\mathrm{Kb}$ deletion of chromosome 22 . The second possibly pathogenic $\mathrm{CNV}$ is a $378 \mathrm{~Kb}$ duplication that involves the distal portion of the 22q11.2 DGS/VCFS region (Patient 8327). A similar duplication was previously reported in a child and father whose cognitive ability was not clearly described [25].

\section{Discussion}

In this study we compared the performance of various AGH systems for the clinical detection of pathogenic CNVs in children with MR. Previous studies that included more limited comparisons of AGH technologies found substantial differences in the CNV detection frequency between platforms, but some of these studies compared much lower resolution techniques and focused on larger CNVs [18] or compared lower- to higher-resolution arrays in an analysis that treated the higher-resolution findings as correct when discrepancy occurred [20]. In addition, most previous comparative studies were retrospective, focusing on the ability of various platforms to identify previously characterized CNVs. These studies only report detection of pathogenic CNVs and do not discuss findings with respect to the more frequent apparently benign variants [18-20].

Here we compared de novo CNVs identified on 3 platforms by analysing each child directly in relationship to his/her parent. This approach was the most cost-efficient to distinguish de novo and inherited CNVs using the comparative AGH methodology. However, using the parents as reference means that half of the hybridizations involve comparisons between samples from different sexes, and copy number estimates involving the $\mathrm{X}$-chromosome(s) requires manual $\mathrm{CNV}$ identification by analysis of raw $\log _{2}$ ratios with the NimbleGen and Affymetrix $500 \mathrm{~K}$ software when there is a sex mismatch.

Each of the three main AGH platforms detected hundreds of autosomal CNVs in these 30 trios - an average of 34 CNVs per trio on NimbleGen arrays, of 22 CNVs on Aglient arrays, and $7 \mathrm{CNVs}$ on Affymetrix $500 \mathrm{~K}$ arrays. What is most striking, however, is that $82 \%$ of the CNV calls were only made on one platform, suggesting a majority of false positive calls. As expected, most of the autosomal CNVs were inherited from one of the parents. However, many of the CNVs called in the child are probably not actually present in the child but rather represent a copy number change in the opposite direction in the parent, e.g., a copy number loss called in the child against the mother but not the father could actually be a copy number gain in the mother that was not transmitted to the child.

Overall, 146 autosomal de novo CNVs and 117 $\mathrm{X}$-chromosomal CNVs were called on the 3 main platforms. 48 (32 de novo autosome and $16 \mathrm{X}$ chromosome) of these CNVs were found on more than one platform. 10 de novo CNVs (9 autosome and $1 \mathrm{X}$ chromosome) were called by all three main platforms. Genotype-phenotype correlations identified $7 \mathrm{CNVs}$ that are likely to be pathogenic and 2 other CNVs that are good 
candidates to cause MR. All 9 of the pathogenic or possibly pathogenic CNVs were identified by each of the three main platforms.

Although we did not fully assess the Illumina Beadchips, their sensitivity appears to be similar to that of the Affymetrix $500 \mathrm{~K}$ arrays. Only 15 trios were assessed on the Affymetrix 6.0 platform. However, it is clear this platform produces many more $\mathrm{CNV}$ calls, although its detection rate for pathogenic and possibly pathogenic CNVs appears to be similar to that of the other SNP arrays. None of the additional de novo CNVs called on the Affymetrix 6.0 platform appears to be pathogenic.

The SMRT array only identified 5 of the 9 CNVs that were thought to be pathogenic or possibly pathogenic. One of the CNVs not identified was only $78 \mathrm{~Kb}$ in size and is probably below the resolution of the SMRT array. It is not clear why the other CNVs were not called by the SMRT array.

A number of differences exist among the platforms studied that may have contributed to the different results. Given the large number of genomic segments that were tested, there is a high probability that some of the de novo CNV calls are false positives in the proband and others are false negatives in a transmitting parent (i.e., the $\mathrm{CNV}$ is actually inherited, rather than de novo, in the proband). Differences in pre-processing, labelling, and hybridization protocols, which were performed according to the various manufacturers' specifications (see Additional File 1), could contribute to the occurrence of false negative and false positive calls. The lowest observed correlation between platforms was for smaller CNVs (data not shown) which highlights the importance of using probe number as a variable for identifying CNVs. Nevertheless, the lack of concordance in every single two-way comparison and the fact that there were de novo CNVs that were identified by one of the platforms that were not identified by any of the others make it very likely that neither optimization of the hybridization conditions nor optimization of the bioinformatic analysis parameters would produce perfect concordance.

Distinguishing pathogenic and benign CNVs is a major part of clinical CNV analysis and goes well beyond the software analysis performed on the data. We performed genotype-phenotype correlations for each de novo CNV using methods similar to those employed clinically, which have recently been discussed at length $[4,12,13,22,23]$. The size cut-off used in our study to assess pathogenicity $(50 \mathrm{~Kb})$ was arbitrary; however, almost all pathogenic CNVs detected by oligonucleotide AGH in recently reported studies of children with MR are much larger than $50 \mathrm{~Kb}[38,39]$.

Although the oligonucleotide or SNP-based AGH technologies studied detected all of the pathogenic or possibly pathogenic CNVs in the $30 \mathrm{MR}$ trios studied, the need to manually assess CNVs on the sex chromosomes when there is a sex-mismatch with the NimbleGen and Affymetrix $500 \mathrm{~K}$ software is an important consideration when testing for MR or other genomic disorders in clinical service laboratories. Our results show that the tiling BAC array is less sensitive than the oligonucleotide or SNP-based arrays studied. In any case, the large number of apparently false positive CNV calls obtained with each of the platforms studied supports the need for validating all such calls with a different methodology before consideration of their possible pathogenicity in a clinical setting.

\section{Conclusions}

It seems unlikely that any of the AGH platforms tested is completely right (or completely wrong) in its $\mathrm{CNV}$ calls. Clinical use of any AGH platform to detect pathogenic CNVs in children with birth defects continues to require considerable skill and experience.

\section{Additional material}

\begin{abstract}
Additional file 1: Technical notes. Detailed protocols for AGH and CNV size comparison between AGH platforms. In addition, there are detailed protocols for CNV validation and brief discussion of the difference between each AGH platform.

Additional file 2: Additional Table 5. Summary of inherited CNVs identified in 30 MR trios identified by the Agilent 244 K, NimbleGen 385 $\mathrm{K}$ and Affymetrix $500 \mathrm{~K}$ arrays and correlation with Illumina Hap550, $32 \mathrm{~K}$ SMRT BAC and Affymetrix 6.0 platforms.

Additional file 3: Additional Table 6. Summary of de novo CNVs identified in 30 MR trios identified by the Agilent 244 K, NimbleGen 385 $\mathrm{K}$ and Affymetrix $500 \mathrm{~K}$ arrays and correlation with Illumina Hap550, $32 \mathrm{~K}$ SMRT BAC and Affymetrix 6.0 platforms.

Additional file 4: Additional Table 7. Summary of all CNV calls made by the Affymetrix 6.0 array in 15 MR trios studied.

Additional file 5: Additional Table 8. Summary of all CNV calls made by the Illumina Hap500 beadchip in 30 MR patients studied.

Additional file 6: Additional Table 9. Summary of all CNV calls made by the $32 \mathrm{~K}$ SMRT BAC tiling path array in 30 MR patients studies.

Additional file 7: Additional Table 10. Summary of all $X$ chromosome CNV in 30 MR trios identified by the Agilent 244 K, NimbleGen $385 \mathrm{~K}$ and Affymetrix $500 \mathrm{~K}$ arrays and correlation with Illumina Hap550, $32 \mathrm{~K}$ SMRT BAC and Affymetrix 6.0 platforms.
\end{abstract}

\section{Acknowledgements}

The authors would like to acknowledge Dr Stéphane LeBihan and Anne Haegart at the Vancouver Prostate Centre Microarray Facility for their assistance with the Agilent array protocol. This work was funded by grants from the Canadian Institutes of Health Research to JMF, the Advocates for thee Rights of Citizens with Developmental Delay (ARC) of Washington State to $P E$, the Réseau de Génétique Médicale Appliquée du Fonds de la Recherche en Santé du Québec (JLM, EL), and by the Fonds d'encouragement à la recherche clinique du CHU Sainte-Justine (JLM, EL). $\mathrm{JM}$ is a Clinician Investigator of the Canadian Institutes of Health Research (Institute of Genetics).

The data resulting from this project have been shared with the research community through publicly accessible Gene Expression Omnibus (GEO) 
under the accession ID GSE27367 http://www.ncbi.nlm.nih.gov/projects/geo/ query/acc.cgi?acc=GSE27367.

\section{Author details}

'Department of Medical Genetics, University of British Columbia, Vancouver, British Columbia, Canada. ${ }^{2}$ McGill University and Genome Quebec Innovation Centre, Montréal, Quebec, Canada. ${ }^{3}$ Children's \& Women's Hospital, Vancouver, British Columbia, Canada. ${ }^{4}$ Genome Sciences Centre, BC Cancer Agency, Vancouver, British Columbia, Canada. ${ }^{5} \mathrm{CHU}$ Sainte-Justine Research Center, Montréal, Quebec, Canada. ${ }^{6}$ British Columbia Cancer Research Centre, Vancouver, British Columbia, Canada. ${ }^{7}$ Child \& Family Research Institute, Vancouver, British Columbia, Canada. ${ }^{8}$ Center of Excellence in Neuromics of Université de Montréal, Montréal, Quebec, Canada. ${ }^{9} \mathrm{CHUM}$ Research Center Montréal, Quebec, Canada.

\section{Authors' contributions}

$\Pi$, AM, and SC and carried out the microarray studies. TT, AM, SC, AD, HQ, DV and JLM analysed the microarray data. TT and AM compared microarray platform data and TT performed the statistical analysis. TT, AM and JMF drafted the manuscript. DC, EM and TT performed molecular analysis to validated CNVs. BC and WLL provided array analysis software. GAR and JLM provided patients. PE, SL, JLM, JFM conceived the study and participated in study design.

\section{Competing interests}

The authors declare that they have no competing interests. All authors read and approved the final manuscript.

Received: 17 June 2010 Accepted: 25 March 2011

Published: 25 March 2011

\section{References}

1. Shaffer LG: American College of Medical Genetics guideline on the cytogenetic evaluation of the individual with developmental delay or mental retardation. Genet Med 2005, 7:650-654.

2. Shaffer LG, Bejjani BA, Torchia B, Kirkpatrick S, Coppinger J, Ballif BC: The identification of microdeletion syndromes and other chromosome abnormalities: cytogenetic methods of the past, new technologies for the future. Am J Med Genet C Semin Med Genet 2007, 145C:335-345.

3. Stankiewicz $P$, Beaudet AL: Use of array $C G H$ in the evaluation of dysmorphology, malformations, developmental delay, and idiopathic mental retardation. Curr Opin Genet Dev 2007, 17:182-192.

4. Zahir F, Friedman JM: The impact of array genomic hybridization on mental retardation research: a review of current technologies and thei clinical utility. Clin Genet 2007, 72:271-287

5. Kidd JM, Cooper GM, Donahue WF, et al: Mapping and sequencing of structural variation from eight human genomes. Nature 2008, 453:56-64.

6. Levy $\mathrm{S}$, Sutton $\mathrm{G}, \mathrm{Ng} P \mathrm{PC}$, et al: The diploid genome sequence of an individual human. PLOS Biol $2007,5 \cdot e 254$

7. Redon R, Ishikawa S, Fitch KR, et al: Global variation in copy number in the human genome. Nature 2006, 444:444-454.

8. Wang J, Wang W, Li R, et al: The diploid genome sequence of an Asian individual. Nature 2008, 456:60-65.

9. Wheeler DA, Srinivasan M, Egholm M, et al: The complete genome of an individual by massively parallel DNA sequencing. Nature 2008, 452:872-876.

10. Friedman JM: High-resolution array genomic hybridization in prenatal diagnosis. Prenat Diagn 2009, 29:20-28.

11. Manning M, Hudgins L: Use of array-based technology in the practice of medical genetics. Genet Med 2007, 9:650-653.

12. Rodriguez-Revenga L, Mila M, Rosenberg C, Lamb A, Lee C: Structural variation in the human genome: the impact of copy number variants on clinical diagnosis. Genet Med 2007, 9:600-606.

13. Vermeesch JR, Fiegler $H$, de Leeuw N, et al: Guidelines for molecular karyotyping in constitutional genetic diagnosis. Eur J Hum Genet 2007, 15:1105-1114

14. Aradhya S, Cherry AM: Array-based comparative genomic hybridization: clinical contexts for targeted and whole-genome designs. Genet Med 2007, 9:553-559

15. Shaikh TH: Oligonucleotide arrays for high-resolution analysis of copy number alteration in mental retardation/multiple congenital anomalies. Genet Med 2007, 9:617-625.
16. Veltman JA, de Vries BB: Whole-genome array comparative genome hybridization: the preferred diagnostic choice in postnatal clinical cytogenetics. J Mol Diagn 2007, 9:277.

17. Zhang ZF, Ruivenkamp C, Staaf J, et al: Detection of submicroscopic constitutional chromosome aberrations in clinical diagnostics: a validation of the practical performance of different array platforms. Eur $J$ Hum Genet 2008, 16:786-792

18. Aston $E$, Whitby $H$, Maxwell $T$, et al: Comparison of targeted and whole genome analysis of postnatal specimens using a commercially available array based comparative genomic hybridisation (aCGH) microarray platform. J Med Genet 2008, 45:268-274.

19. Hehir-Kwa JY, Egmont-Petersen M, Janssen IM, Smeets D, van Kessel AG, Veltman JA: Genome-wide copy number profiling on high-density bacterial artificial chromosomes, single-nucleotide polymorphisms, and oligonucleotide microarrays: a platform comparison based on statistical power analysis. DNA Res 2007, 14:1-11.

20. Wicker N, Carles A, Mills IG, et al: A new look towards BAC-based array $\mathrm{CGH}$ through a comprehensive comparison with oligo-based array $\mathrm{CGH}$. BMC Genomics 2007, 8:84.

21. de Vries BB, White SM, Knight SJ, et al: Clinical studies on submicroscopic subtelomeric rearrangements: a checklist. J Med Genet 2001, 38:145-150.

22. Carter NP: Methods and strategies for analyzing copy number variation using DNA microarrays. Nat Genet 2007, 39:S16-21.

23. Lee C, lafrate AJ, Brothman AR: Copy number variations and clinical cytogenetic diagnosis of constitutional disorders. Nat Genet 2007, 39: S48-54.

24. Brunetti-Pierri N, Berg JS, Scaglia F, et al: Recurrent reciprocal 1q21.1 deletions and duplications associated with microcephaly or macrocephaly and developmental and behavioral abnormalities. Nat Genet 2008, 40:1466-1471.

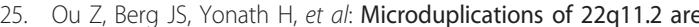
frequently inherited and are associated with variable phenotypes. Genet Med 2008, 10:267-277.

26. Rosenberg C, Knijnenburg J, Bakker E, et al: Array-CGH detection of micro rearrangements in mentally retarded individuals: clinical significance of imbalances present both in affected children and normal parents. J Med Genet 2006, 43:180-186.

27. van Bon BW, Mefford HC, Menten B, et al: Further delineation of the $15 q 13$ microdeletion and duplication syndromes: a clinical spectrum varying from non-pathogenic to a severe outcome. J Med Genet 2009, 46:511-523.

28. Dumitrescu AM, Liao XH, Best TB, Brockmann K, Refetoff S: A novel syndrome combining thyroid and neurological abnormalities is associated with mutations in a monocarboxylate transporter gene. Am J Hum Genet 2004, 74:168-175.

29. Shapiro LJ, Yen P, Pomerantz D, Martin E, Rolewic L, Mohandas T: Molecular studies of deletions at the human steroid sulfatase locus. Proc Natl Acad Sci USA 1989, 86:8477-8481.

30. Dibbens LM, Tarpey PS, Hynes K, et al: X-linked protocadherin 19 mutations cause female-limited epilepsy and cognitive impairment. $\mathrm{Na}$ Genet 2008, 40:776-781.

31. Tarpey P, Parnau J, Blow M, et al: Mutations in the DLG3 gene cause nonsyndromic X-linked mental retardation. Am J Hum Genet 2004, 75:318-324.

32. Kleefstra T, Koolen DA, Nillesen WM, et al: Interstitial $2.2 \mathrm{Mb}$ deletion at $9 q 34$ in a patient with mental retardation but without classical features of the 9q subtelomeric deletion syndrome. Am J Med Genet A 2006, 140:618-623.

33. Koolen DA, Sharp AJ, Hurst JA, et al: Clinical and molecular delineation of the 17q21.31 microdeletion syndrome. J Med Genet 2008, 45:710-720.

34. Rauch A, Zink S, Zweier C, et al: Systematic assessment of atypical deletions reveals genotype-phenotype correlation in 22q11.2. J Med Genet 2005, 42:871-876.

35. Schorry EK, Keddache M, Lanphear N, et al: Genotype-phenotype correlations in Rubinstein-Taybi syndrome. Am J Med Genet A 2008, 146A:2512-2519.

36. Patel KG, Liu C, Cameron PL, Cameron RS: Myr 8, a novel unconventional myosin expressed during brain development associates with the protein phosphatase catalytic subunits 1alpha and 1gamma1. J Neurosci 2001, 21:7954-7968. 
37. Thomas S, Ritter B, Verbich D, et al: Intersectin regulates dendritic spine development and somatodendritic endocytosis but not synaptic vesicle recycling in hippocampal neurons. J Biol Chem 2009, 284:12410-12419.

38. Bruno DL, Ganesamoorthy D, Schoumans J, et al: Detection of cryptic pathogenic copy number variations and constitutional loss of heterozygosity using high resolution SNP microarray analysis in 117 patients referred for cytogenetic analysis and impact on clinical practice. J Med Genet 2009, 46:123-131.

39. Fan YS, Jayakar $P$, Zhu H, et al: Detection of pathogenic gene copy number variations in patients with mental retardation by genomewide oligonucleotide array comparative genomic hybridization. Hum Mutat 2007, 28:1124-1132.

40. Moehle C, Ackermann N, Langmann T, et al: Aberrant intestinal expression and allelic variants of mucin genes associated with inflammatory bowel disease. J Mol Med 2006, 84:1055-1066.

41. Ogino S, Wilson RB: Spinal muscular atrophy: molecular genetics and diagnostics. Expert Rev Mol Diagn 2004, 4:15-29.

42. Shibui A, Tsunoda T, Seki N, Suzuki Y, Sugano S, Sugane K: Cloning, expression analysis, and chromosomal localization of a novel butyrophilin-like receptor. J Hum Genet 1999, 44:249-252.

43. Azen EA, Maeda N: Molecular genetics of human salivary proteins and their polymorphisms. Adv Hum Genet 1988, 17:141-199.

44. Garcia-Minaur S, Fantes J, Murray RS, et al: A novel atypical 22q11.2 distal deletion in father and son. J Med Genet 2002, 39:E62.

45. Alvarez E, Zhou W, Witta SE, Freed CR: Characterization of the Bex gene family in humans, mice, and rats. Gene 2005, 357:18-28.

46. Bassi MT, Ramesar RS, Caciotti B, et al: X-linked late-onset sensorineural deafness caused by a deletion involving OA1 and a novel gene containing WD-40 repeats. Am J Hum Genet 1999, 64:1604-1616.

47. Chomez P, De Backer O, Bertrand M, De Plaen E, Boon T, Lucas S: An overview of the MAGE gene family with the identification of all human members of the family. Cancer Res 2001, 61:5544-5551.

48. Yen PH, Ellison J, Salido EC, Mohandas T, Shapiro L: Isolation of a new gene from the distal short arm of the human $X$ chromosome that escapes X-inactivation. Hum Mol Genet 1992, 1:47-52.

49. Miyake A, Konishi M, Martin FH, et al: Structure and expression of a novel member, FGF-16, on the fibroblast growth factor family. Biochem Biophys Res Commun 1998, 243:148-152.

50. Lurquin C, De Smet C, Brasseur F, et al: Two members of the human MAGEB gene family located in Xp21.3 are expressed in tumors of various histological origins. Genomics 1997, 46:397-408.

\section{Pre-publication history}

The pre-publication history for this paper can be accessed here: http://www.biomedcentral.com/1755-8794/4/25/prepub

doi:10.1186/1755-8794-4-25

Cite this article as: Tucker et al:: Comparison of genome-wide array genomic hybridization platforms for the detection of copy number variants in idiopathic mental retardation. BMC Medical Genomics 2011 $4: 25$.

\section{Submit your next manuscript to BioMed Central and take full advantage of:}

- Convenient online submission

- Thorough peer review

- No space constraints or color figure charges

- Immediate publication on acceptance

- Inclusion in PubMed, CAS, Scopus and Google Scholar

- Research which is freely available for redistribution 